Asia-Pac. J. Atmos. Sci., 52(5), 519, 2016

DOI:10.1007/s13143-016-0044-6

ERRATUM

\title{
Erratum to: Climate Change Projections over India by a Downscaling Approach Using PRECIS
}

Prasanta Kumar Bal ${ }^{1,2}$, Andimuthu Ramachandran ${ }^{1}$, Kandasamy Palanivelu ${ }^{1}$, Perumal Thirumurugan ${ }^{1}$, Rajadurai Geetha ${ }^{1}$, and Balakrishnan Bhaskaran ${ }^{3}$

${ }^{1}$ Centre for Climate Change and Adaptation Research, Anna University, Chennai, India

${ }^{2}$ National Centre for Medium Range Weather Forecasting, Ministry of Earth Science, Noida, India

${ }^{3}$ Fujitsu Laboratories of Europe Limited, Hayes Park, Middlesex, UK

(C) The Korean Meteorological Society and Springer 2016

Erratum to: Asia-Pac. J. Atmos. Sci., 52(4), 353-369, 2016

DOI:10.1007/s13143-016-0004-1

The author "Bhaski Bhaskaran" and his affiliation "Fujitsu Laboratory of Europe, Middlesex, UK" should be replaced by "Balakrishnan Bhaskaran", "Fujitsu Laboratories of Europe Limited, Hayes Park, Middlesex, UK", respectively.

The corrected name and affiliation are shown in this erratum.

Corresponding Author: Prasanta Kumar Bal, Centre for Climate Change and Adaptation Research, Anna University, Chennai 600025 , India.

E-mail: prasantbal.cc@gmail.com 